\title{
Relationship between Physical Examinations and Two-Dimensional Computed Tomographic Findings in Children with Intoeing Gait
}

\author{
Hyun Dong Kim, M.D., Dong Seok Lee, M.D., Mi Ja Eom, M.D., Ji Sun Hwang, M.D., \\ Na Mi Han, M.D., Geun Yeol Jo, M.D. ${ }^{1}$
}

Department of Physical Medicine and Rehabilitation, Pusan Paik Hospital, Busan 614-735,

${ }^{1}$ Haeundae Paik Hospital, Inje University College of Medicine, Busan 612-030, Korea

\begin{abstract}
Objective To evaluate the validity of physical examinations by assessment of correlation between physical examinations and CT measurements in children with intoeing gait and the causes of intoeing gait by age using CT measurements.

Method Twenty-six children with intoeing gait participated in this study. The internal and external hip rotation, thigh-foot angle and transmalleolar angle were measured. In addition, femoral anteversion and tibial torsion of the subjects were assessed using a CT scan. The measurements of torsional angles were performed twice by two raters. The correlation coefficients between physical examinations and CT measurements were calculated using Pearson correlation. The data was analyzed statistically using SPSS v12.0.

Results The correlation coefficients between physical examinations and CT measurements were not high. Before 5 years of age, intoeing gait was caused by femoral anteversion in $17.86 \%$, tibial torsion in $32.29 \%$ and the combination of causes in $35.71 \%$ of cases. After 6 years of age, the contributions changed to $29.17 \%, 8.33 \%$ and $45.83 \%$, respectively.

Conclusion Before 5 years of age, the common cause of an intoeing gait was tibial torsion, whereas after 6 years of age it was femoral anteversion. Regardless of age, the most common cause of intoeing gait was a combination of causes. This study shows poor correlation between physical examinations and CT. Therefore, it is limiting to use physical examination only for evaluating the cause of intoeing gait in clinical practice.
\end{abstract}

Key Words Intoeing gait, Femoral anteversion, Tibial torsion, Thigh foot angle, Hip internal rotation

Received July 29, 2010; Accepted February 28, 2011

Corresponding author: Dong Seok Lee

Department of Physical Medicine and Rehabilitation, Pusan Paik Hospital, Inje University College of Medicine, 633-165, Gaegeum-dong, Busanjin-gu, Busan 614-735, Korea

Tel: +82-51-890-6754, Fax: +82-51-891-1430, E-mail: ddongsiki@naver. com

(c) This is an open-access article distributed under the terms of the Creative Commons Attribution Non-Commercial License (http://creativecommons.org/ licenses/by-nc/3.0) which permits unrestricted noncommercial use, distribution, and reproduction in any medium, provided the original work is properly cited.

Copyright $\odot 2011$ by Korean Academy of Rehabilitation Medicine

\section{INTRODUCTION}

To identify causes including femoral anteversion and tibial internal torsion in children with intoeing gait, physical examination, X-ray, ultrasound, computed tomography (CT) scan, and magnetic resonance imaging (MRI) have been used in the clinical setting. ${ }^{1,2}$ The most commonly used methods in clinical practice are physical examinations and CT scan. The reliability and 
validity of CT scans for causal analysis of intoeing gait in many studies has been proven, but the validity of physical examinations as an evaluation tool has not yet been demonstrated. ${ }^{3-8}$ However, to conduct a CT scan for every child with intoeing gait or to monitor each stage of progress is too costly. Thus, this study attempted to determine the correlation between the commonly used physical examinations in clinical practice and CT measurements in evaluating children who came to the hospital with intoeing gait and whether the physical examination was valid to replace CT scan. Moreover, this study intended to evaluate the causes of intoeing gait by age and by classifying them into femoral anteversion and tibial torsion.

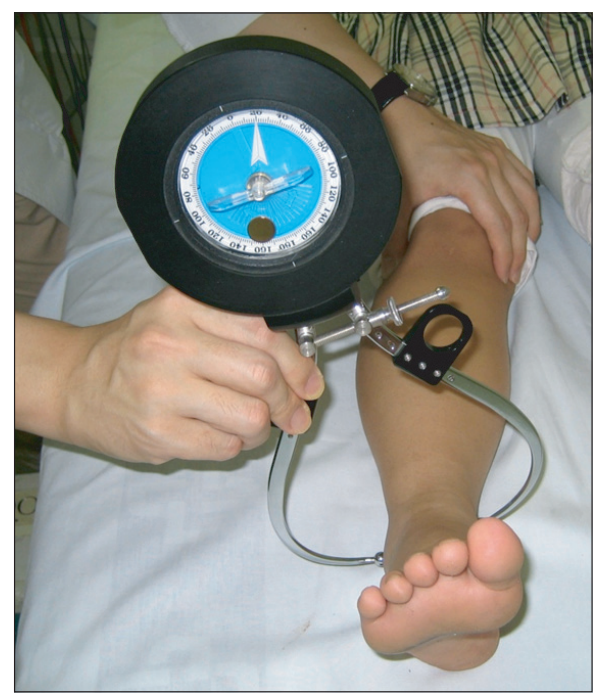

Fig. 1. Gravity goniometer.
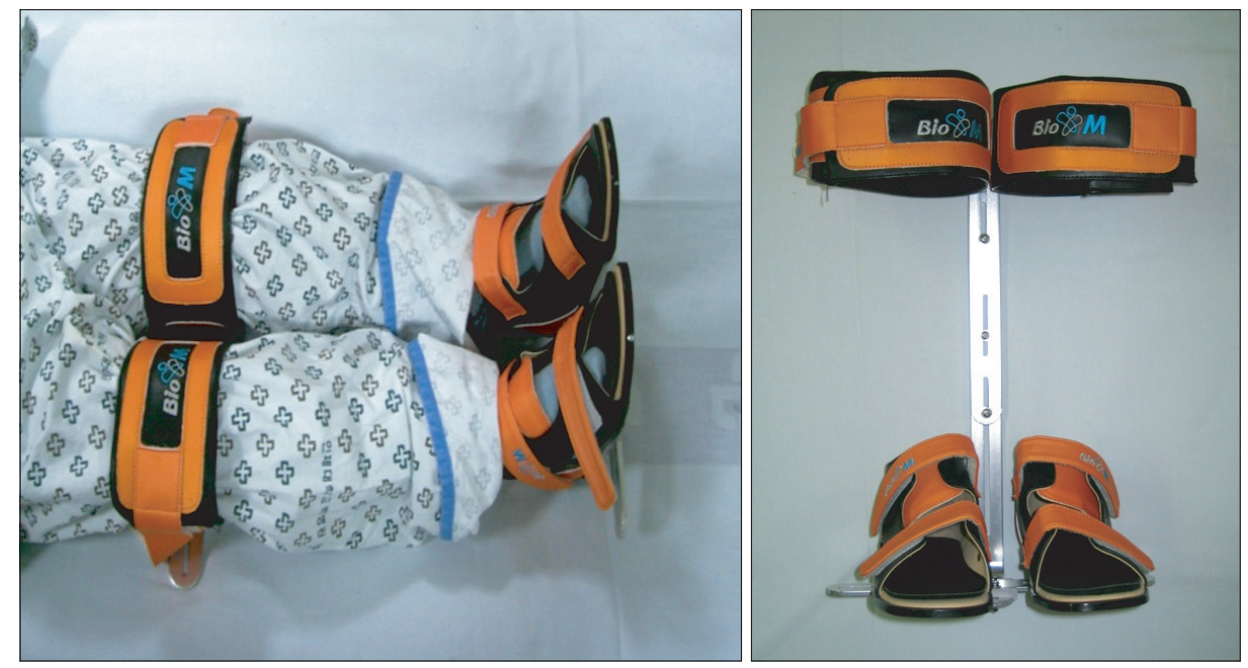

Fig. 2. Lower extremities fixation brace was used for evaluation of intoeing gait children by CT. 
The transmalleolar angle was measured using a gravity goniometer in the supine position after marking the medial malleolus of the tibia and lateral maleoulus of the fibula while extending the knees to the coronal plane (Fig. 1). ${ }^{10}$

CT scan was used to measure femoral anteversion and tibial torsion. Femoral anteversion was measured by the angle between the two lines with the line that connected the medial and lateral distal femur as the primary axis and the one that passed from the femoral head to femoral neck as the axis of proximal femur (Fig. 2). ${ }^{6}$

Tibial torsion was obtained by measuring the angle between the line that connected the widest section of tibial intercondylar distances with the intercondylar distance behind the tibia as the primary axis and the one vertical to the rear and front diameter of the tibia from the lowest tibia and passed the center of the lateral malleolus of the fibula (Fig. 3).$^{6-8,11-13}$ A specially devised tibial holder was used to reduce errors when the children moved during the CT scan or showed different positions of their lower limb (Fig. 4).

The causal analysis of intoeing gait by age was conducted by comparing femoral anteversion and tibial torsion obtained by CT measurements with the data of normal children by age, which was then divided into normality and abnormality. ${ }^{6,14}$

Femoral anteversion was based on the age-specific normal values of the studies of Billing ${ }^{14}$ If the angle was bigger than the standard, it became one of the causes of intoeing gait. As tibial torsion had no age-specific classifications, it was divided into tibial internal torsion if the angle had a lower value than the standard $40^{\circ} \pm 9$ according to the studies of Jend et al. ${ }^{6}$ and as a cause of intoeing gait.
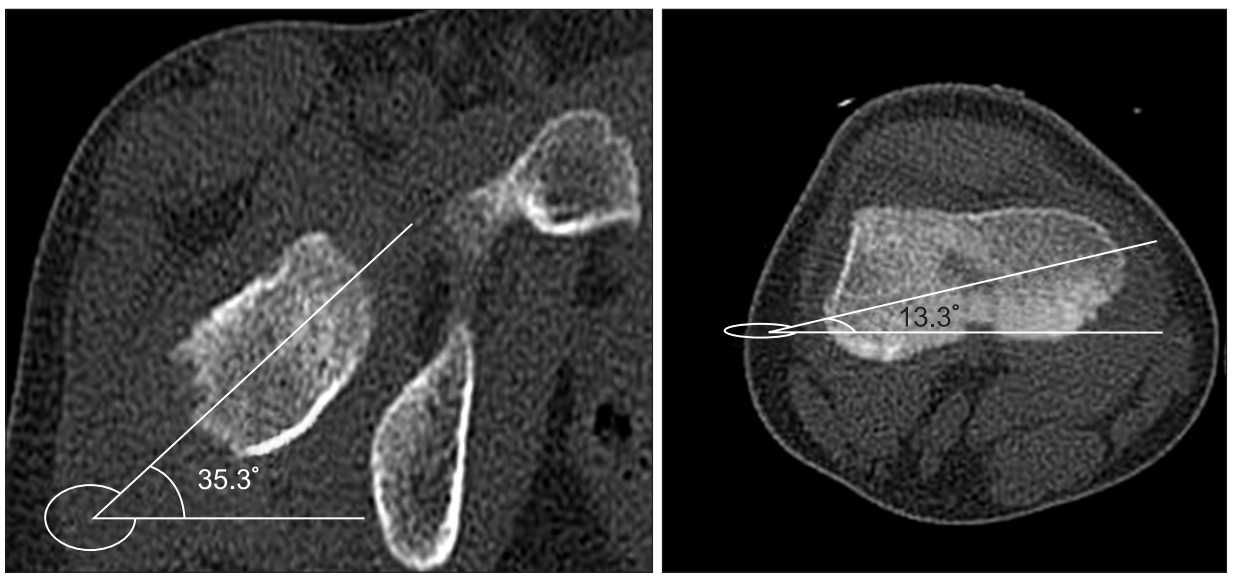

Fig. 3. The reference lines are the bisection line of femoral head and neck and the line of femoral condyles as described by Weiner et al. ${ }^{8}$ The angle between these two planes show femoral anteversion.
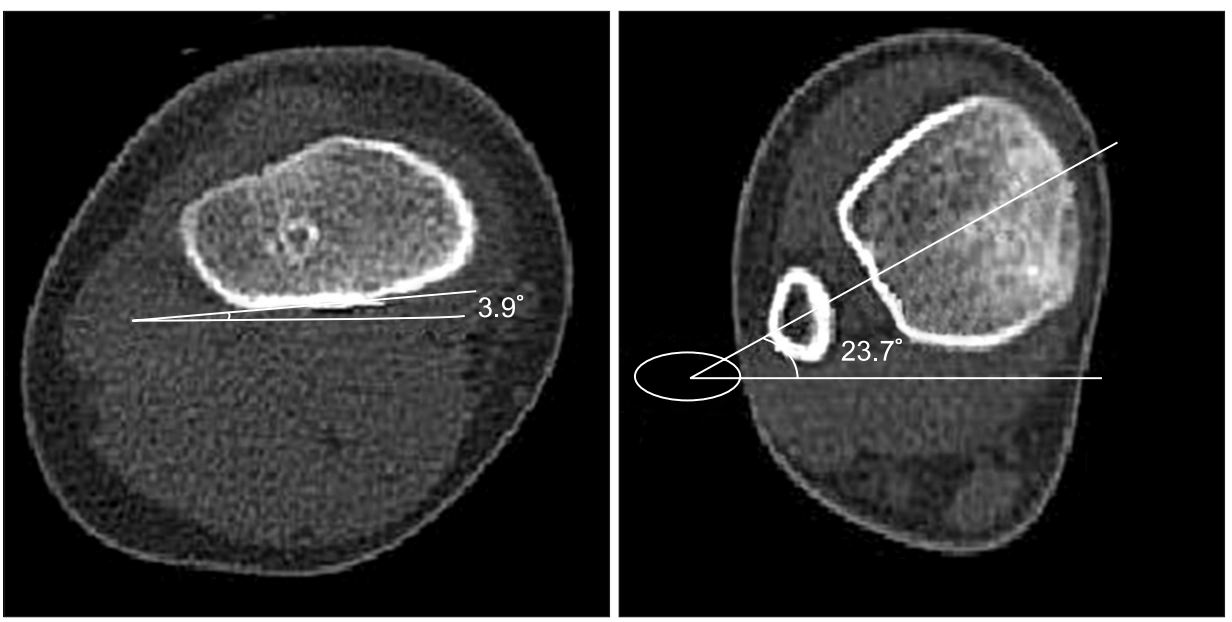

Fig. 4. The reference line is the posterior border of the proximal tibial plateau, and the bisection line of the distal tibial plateau through the center of the fibula. The angle of these two line results in tibial torsion. 


\section{Data analysis}

Two skilled doctors in the department conducted physical examinations to analyze the interobservers reliability. The mean value was used as the data value for evaluation and comparison by CT scan.

The two doctors in the department of Physical Medicine and Rehabilitation measured CT findings twice with an interval of one week respectively and analyzed the reliability of interobservers and intraobservers. The mean value for the 4 measurement values was used as the data value.

The correlation between femoral anteversion measured by CT scan and hip internal rotation and external rotation measured by physical examinations was analyzed. Additionally, the correlation between tibial torsion measured by CT scan and physically examined thigh-foot angle and transmalleolar angle was analyzed using Pearson's correlation coefficients at a significance level of $p<0.05$. Data was processed statistically using SPSS 12.0 for Windows (SPSS Inc., Chicago, IL, USA).

\section{RESULTS}

The findings were obtained for the 52 left and right feet in 26 children with intoeing gait. The mean value of hip internal rotation, external rotation, thigh-foot angle and transmalleolar angle after physical examinations was $63.28^{\circ} \pm 10.53,49.13^{\circ} \pm 8.47,3.68^{\circ} \pm 4.84$, and $20.80^{\circ} \pm 5.20$, respectively, while the value of femoral anteversion and tibial torsion after CT scan was $25.11 \pm 9.41$ and $26.01 \pm 8.62$, respectively (Table 1 ).

The interobservers reliability for hip internal rotation, external rotation, thigh-foot angle and transmalleolar angle was $0.739,0.824,0.861$, and 0.801 , respectively (Table 2).
Interoberservers and intraobserver reliabilities for femoral anteversion angle and tibial torsion angle by CT measurements ranged from 0.968 to 0.991 and from 0.984

Table 2. Interobserver Reliablilities of Physical Examination

\begin{tabular}{ccc}
\hline $\begin{array}{c}\text { Physical } \\
\text { examination }\end{array}$ & $\begin{array}{c}\text { Correlation } \\
\text { coefficient }\end{array}$ & $\begin{array}{c}\text { 95\% confidence } \\
\text { interval }\end{array}$ \\
\hline HIR & 0.739 & $0.597-0.841$ \\
HER & 0.824 & $0.731-0.895$ \\
TFA & 0.861 & $0.770-0.917$ \\
TMA & 0.801 & $0.678-0.881$ \\
\hline
\end{tabular}

HIR: Hip internal rotation, HER: Hip external rotation, TFA: Thigh-foot angle, TMA: Transmalleoular angle

Table 3. Intraobserver and Interobserver Reliablilities of CT Measurement

\begin{tabular}{|c|c|c|c|c|}
\hline & Reliability & Rater & $\begin{array}{c}\text { Correlation } \\
\text { coefficient }\end{array}$ & $\begin{array}{c}95 \% \\
\text { confidence } \\
\text { interval }\end{array}$ \\
\hline \multirow[t]{4}{*}{ FA } & Intraobserver & First rater & 0.989 & $0.982-0.994$ \\
\hline & & $\begin{array}{l}\text { Second } \\
\text { rater }\end{array}$ & 0.973 & $0.953-0.984$ \\
\hline & Interobserver & $\begin{array}{l}\text { First } \\
\text { session }\end{array}$ & 0.991 & 0.985-0.995 \\
\hline & & $\begin{array}{l}\text { Second } \\
\text { session }\end{array}$ & 0.968 & $0.944-0.981$ \\
\hline \multirow[t]{4}{*}{ TT } & Intraobserver & First rater & 0.990 & $0.983-0.994$ \\
\hline & & $\begin{array}{l}\text { Second } \\
\text { rater }\end{array}$ & 0.984 & $0.972-0.991$ \\
\hline & Interobserver & $\begin{array}{l}\text { First } \\
\text { session }\end{array}$ & 0.989 & $0.981-0.994$ \\
\hline & & $\begin{array}{l}\text { Second } \\
\text { session }\end{array}$ & 0.989 & $0.981-0.994$ \\
\hline
\end{tabular}

FA: Femoral anteversion, TT: Tibial torsion

Table 1. Description of Subjects

\begin{tabular}{llcrrrr}
\hline Exam. & N & Range & Min. & Max. & Mean & SD \\
\hline HIR & 52 & 43.50 & 42.50 & 86.00 & 63.28 & 10.53 \\
HER & 52 & 30.50 & 37.50 & 68.00 & 49.13 & 8.47 \\
TFA & 52 & 31.50 & -13.50 & 18.00 & 3.68 & 4.84 \\
TMA & 52 & 25.50 & 13.50 & 39.00 & 20.80 & 5.20 \\
FA & 52 & 43.47 & 7.73 & 52.20 & 25.11 & 9.41 \\
TT & 52 & 33.43 & 8.50 & 41.93 & 26.01 & 8.62 \\
\hline
\end{tabular}

HIR: Hip internal rotation, HER: Hip external rotation, TFA: Thigh-foot angle, TMA: Transmalleoular angle, FA: Femoral anteversion, TT: Tibial torsion 
to 0.990 , respectively (Table 3).

For the correlation between physical examinations and CT measurements, hip internal rotation and external rotation was compared with femoral anteversion, while thigh-foot angle and transmalleolar angle was compared

Table 4. Correlations between Physical Examinations and CT Measurements

\begin{tabular}{cccc}
\hline $\begin{array}{c}\text { Physical } \\
\text { examination }\end{array}$ & CT measurement & $\begin{array}{c}\text { Correlation } \\
\text { coefficient (r) }\end{array}$ & p-value \\
\hline HIR & FA & 0.31 & 0.25 \\
HER & FA & 0.17 & 0.23 \\
TFA & TT & 0.14 & 0.31 \\
TMA & TT & 0.43 & 0.00 \\
\hline
\end{tabular}

HIR: Hip internal rotation, HER: Hip external rotation, TFA: Thigh-foot angle, TMA: Transmalleoular angle, FA: Femoral anteversion, TT: Tibial torsion
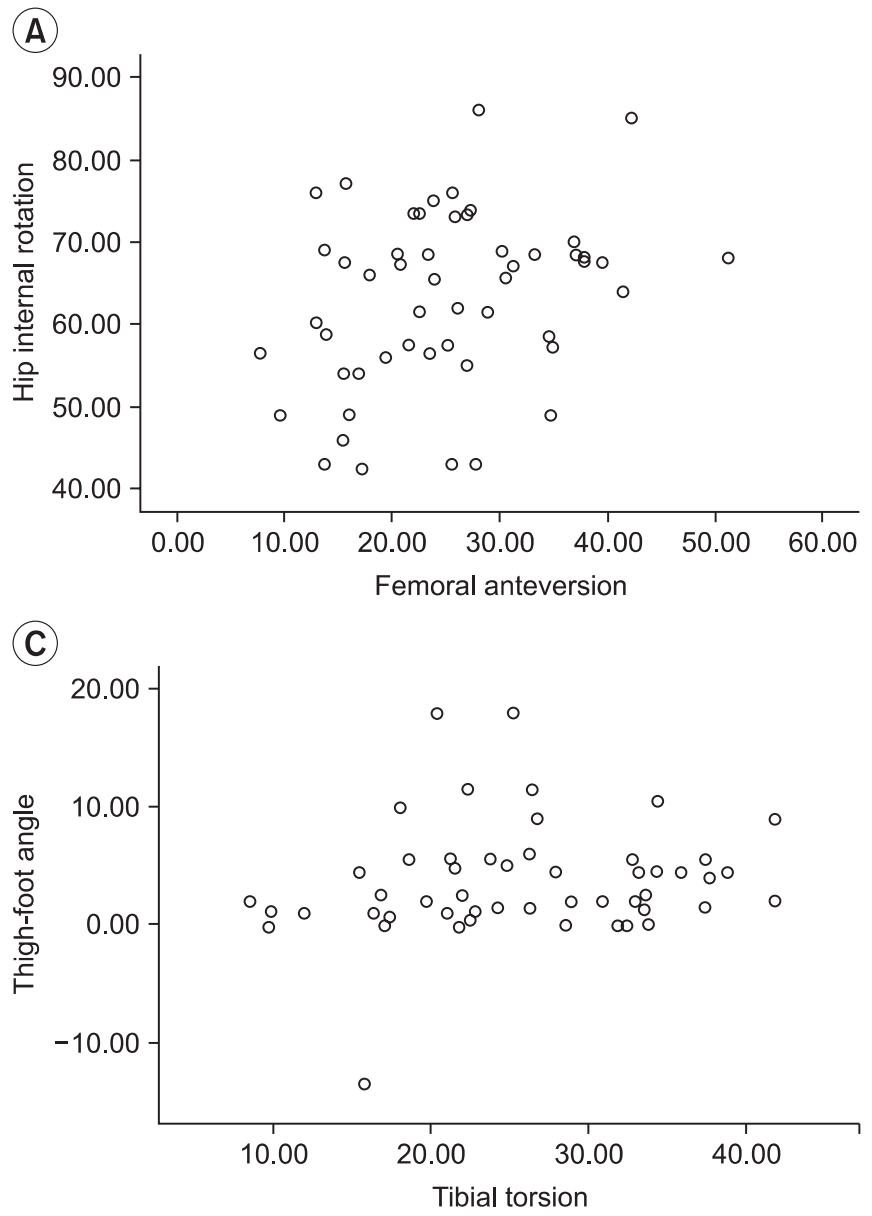

with tibial torsion. The correlation was $0.31,0.17,0.14$, and 0.43 , respectively (Table 4, Fig. 5). While tibial torsion and transmalleolar angle showed a moderate correlation, the correlation between the rests of examinations was low. The low correlation in individual data was demonstrated as femoral anteversion angle that varied within the range of $16.5^{\circ}$ and $52.6^{\circ}$ for children with identical $70^{\circ}$ of hip internal rotation.

The cause of intoeing gait by age showed that comparatively more children under the age of 5 were generally accompanied by tibial internal torsion, while comparatively more children over the age of 6 were accompanied by femoral anteversion generally. Several cases were accompanied by both (Table 5 ).

For children under 5, 17.86\% (5 feet/28 feet) had femoral anteversion only, while $39.29 \%$ ( 11 feet $/ 28$ feet) had tibial internal torsion only and $35.71 \%$ (10 feet/28 feet) had
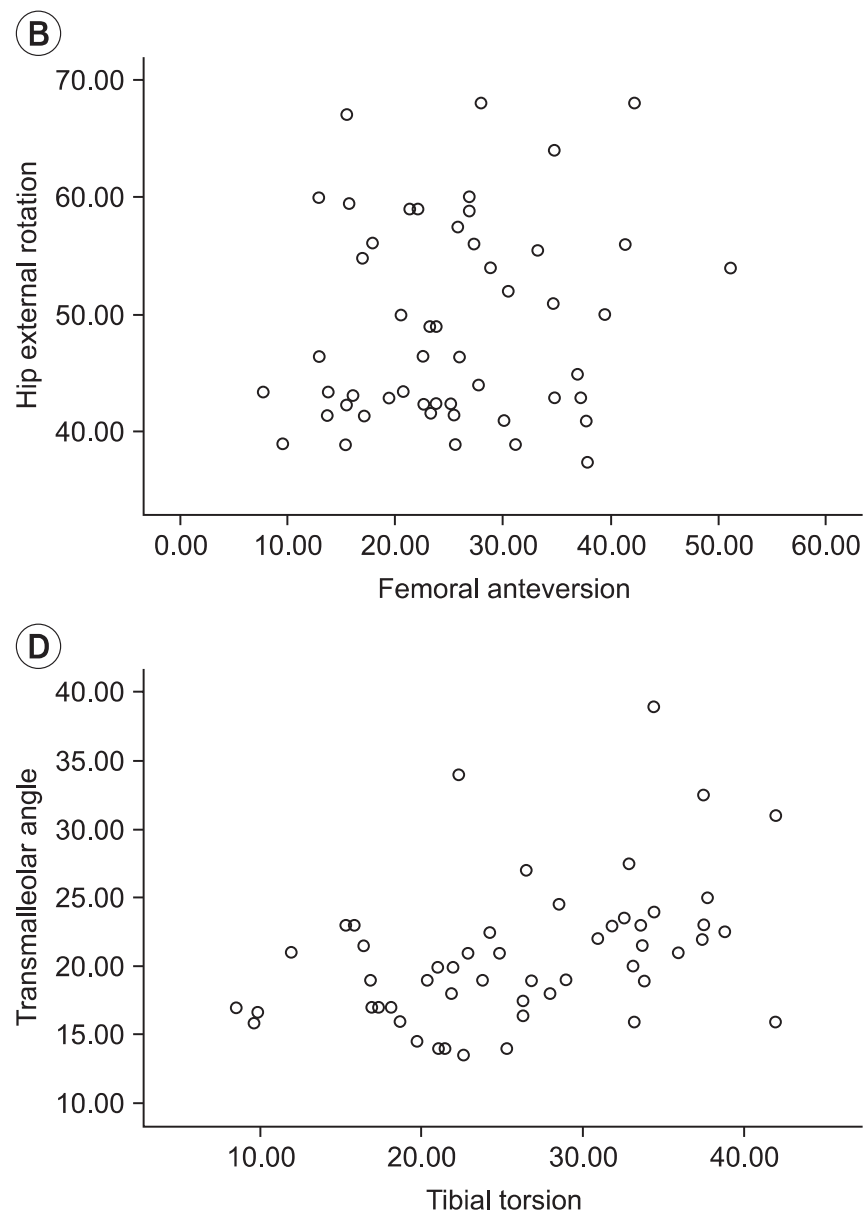

Fig. 5. Scatter plot of relationship between physical examinations and CT measurements (A: relationship between femoral anteversion and hip internal rotation, B: relationship between femoral anteversion and hip external rotation, C: relationship between tibial torsion and thigh-foot angle, D: relationship between tibial torsion and transmalleolar angle). 
Table 5. The Causes of Intoeing Gait by the Age of the Children

\begin{tabular}{cccccc}
\hline Age & $\begin{array}{c}\text { Number of } \\
\text { patient }\end{array}$ & $\begin{array}{c}\text { Abnormal } \\
\text { FA frequency (\%) }\end{array}$ & $\begin{array}{c}\text { Abnormal TT } \\
\text { frequency (\%) }\end{array}$ & $\begin{array}{c}\text { Abnormal FA and } \\
\text { TT (\%) }\end{array}$ & $\begin{array}{c}\text { Normal FA and TT } \\
(\%)\end{array}$ \\
\hline 2 & 8 & $2(25)$ & $3(37.5)$ & $1(12.5)$ & $2(25)$ \\
3 & 4 & $1(25)$ & $0(0)$ & $3(75)$ & $0(0)$ \\
\hline 4 & 10 & $2(20)$ & $5(50)$ & $3(30)$ & $0(0)$ \\
\hline 5 & 6 & $0(0)$ & $3(50)$ & $3(50)$ & $0(0)$ \\
6 & 6 & $0(0)$ & $0(0)$ & $4(66.7)$ & $2(33.3)$ \\
\hline 7 & 2 & $0(0)$ & $0(0)$ & $2(100)$ & $0(0)$ \\
\hline 8 & 2 & $1(50)$ & $0(0)$ & $1(50)$ & $0(0)$ \\
\hline 9 & 6 & $2(33.3)$ & $2(33.3)$ & $0(0)$ & $2(33.3)$ \\
10 & 4 & $2(50)$ & $0(0)$ & $2(50)$ & $0(0)$ \\
11 & 2 & $2(100)$ & $0(0)$ & $0(0)$ & $0(0)$ \\
18 & 2 & $0(0)$ & $0(0)$ & $2(100)$ & $0(0)$ \\
\hline
\end{tabular}

FA: Femoral anteversion, TT: Tibial torsion

both and $7.14 \%$ ( 2 feet/28 feet) had normal feet. Children over 6 showed $29.17 \%$ ( 7 feet/24 feet), 8.33\% ( 2 feet $/ 24$ feet), $45.83 \%$ (11 feet/24 feet), and $16.67 \%$ ( 4 feet $/ 24$ feet), respectively.

\section{DISCUSSION}

This study aimed at assessing the correlation between tests after performing CT scans and physical examinations for children who visit the hospital with intoeing gait as the chief complaint. It also focused on identifying the cause of intoeing gait by age.

According to Rethlefsen et al., ${ }^{15}$ intoeing gait can be defined to show over $0^{\circ}$ of the foot progression angle to point inward in more than $50 \%$ of the walking cycle. In this study, children whose feet were coated with white chalk were asked to walk straight towards their parents on a straight line to measure the foot progression angle in compliance with the studies of Sass and Hassan. ${ }^{16}$ The children were divided into a group of intoeing gait using this method and had physical examinations and CT scan.

CT measurements assessed tibial internal torsion and femoral anteversion, and equivalent physical examinations assessed thigh-foot angle and transmalleolar angle corresponding to tibial internal torsion and hip internal rotation and external rotation corresponding to femoral anteversion. The comparative analysis of the correlation in this study ${ }^{3-8}$ was based on the validity of CT measurements for tibial internal torsion and femoral anteversion proven in a number of studies including one using a cadaver. ${ }^{4,5}$

The correlation between CT measurements and physical examination findings showed a moderate correlation between tibial torsion angle and transmalleolar angle, whereas correlation for the rest of the examinations was low. This suggested CT measurements and physical examinations reflected different parts to each other.

While femoral anteversion angle shows torsion between the femoral head and distal femur, hip internal rotation and external rotation not only mirror torsion between the femoral head and femur but also the inclination of femoral head to the acetabulum. To review the measuring of femoral anteversion angle using CT scan, the angle between the two lines was measured with the line that connected the medial and lateral distal femur as the primary axis and the one that passed from the femoral head to the femoral neck as the axis of proximal femur. The axis of proximal femur here is the line that passed the femoral neck, and the angle between the pelvis and femoral head was not considered. This shows that the correlation between the examinations should be low because internal rotation and external rotation would be out of the normal range depending on the femoral head angle although femoral anteversion angle is normal.

The low correlation between tibial torsion and the thighfoot angle is because the distal tibia for tibial torsion measures the lowest tibia it is impossible to reflect the feet condition. However, the standard line of distal tibia for the thigh-foot angle is the sole and reflects the feet condition, whereas the transmalleolar angle measured 
from the ankle relatively has no reflection of the feet condition. For this reason, its correlation with tibial torsion is the relatively higher moderate level. It implies that children with a deformed foot should be evaluated not with the thigh-foot angle but with the transmalleolar angle. ${ }^{1}$

In summary, it is interpreted that hip internal rotation and external rotation cannot reflect femoral anteversion adequately, and although thigh-foot angle cannot mirror tibial torsion properly, transmalleolar angle reflects the tibial torsion angle to a certain degree.

This implies that the physical examinations as a test to assess the cause of intoeing gait compared to CT measurements were less significant but the low correlation between hip internal rotation and external rotation by physical examinations and femoral anteversion assessed by CT scans leading to low validity of physical examinations as an alternative test, as well as the low validity of thigh-foot angles to tibial torsion.

The reliability of physical examinations was also lower than CT measurements. As shown in Table 3, while the intraclass correlation coefficient interobservers or intraobserver were all over 0.95 in CT measurements, they were $0.739,0.824,0.861$, and 0.801 for hip internal rotation, external rotation, thigh-foot angle and transmalleolar angle, respectively, in physical examinations. This can be attributed to the CT scan having fewer errors than physical examinations and offering more accurate measurement findings.

Intoeing gait can result from various causes from the femur to the foot. The causes are commonly divided into femoral anteversion, tibial internal torsion, and metatarsus adductus. ${ }^{1,16}$ Metatarsus adductus is most common in infants, while tibial internal torsion is common until age 2 , and femoral anteversion after age $3 ., 16,17$

The children who participated in this study were between the age of 2 and 18, and had no abnormal cases against metatarsus adductus reported. The analytical findings of femoral anteversion and tibial internal torsion using a CT scan showed that the distinction between femoral anteversion and tibial internal torsion by age as the cause was not clear. Many cases of both femoral anteversion and tibial internal torsion were included within the abnormal range. Furthermore, while intoeing gait by tibial internal torsion only was $50 \%$ for 4 and also $50 \%$ for 5 for children at the age of 4 and 5 , intoeing gait by femoral anteversion was $20 \%$ and $0 \%$, respectively, indicating the relatively low rate. For children at the age of 10 and 11, intoeing gait by femoral anteversion was $50 \%$ and $100 \%$, respectively, whereas intoeing gait by tibial internal torsion was $0 \%$ for both ages. Overall, the rate of tibial internal torsion was observed high in children under the age of 5 , while the rate of femoral anteversion was mildly prevalent in children over 6 (Table 5). These findings were different from previous studies. ${ }^{1,16,17}$ Femoral anteversion was known to be the primary cause of intoeing gait for children over 3 years of age, whereas in this study it was the primary cause for children over 6 years of age. This can be explained by the contribution of a large portion of patients who had both tibial internal torsion and femoral anteversion simultaneously, and the small number of total subjects including 4 children ( 8 feet) at 2 years of age which made it difficult to group them by age for children over 2 and 3 years of age. However, this study showed that intoeing gait in children can be caused by a combination of causes.

\section{CONCLUSION}

This study showed a low correlation between classical physical examinations and CT measurements. This could have resulted from the combination of causes of intoeing gait. Therefore, it is recommended to combine physical examinations with CT scans to examine intoeing gait to complement each other. Further studies should include the development of valid physical examinations to substitute CT measurements by evaluating intoeing gait. It is also necessary to evaluate a greater number of patients for a causal analysis of intoeing gait by age in future studies.

\section{REFERENCES}

1. Li YH, Leong JC. Intoeing gait in children. Hong Kong Med J 1999; 5: 360-366

2. Inan M, Altintas F, Duru I. The evaluation and management of rotational deformity in cerebral palsy. Acta Orthop Traumatol Turc 2009; 43: 106-112

3. Butler-Manuel PA, Guy RL, Heatley FW. Measurement of tibial torsion--a new technique applicable to ultrasound and computed tomography. Br J Radiol 1992; 
65: $119-126$

4. Clementz BG, Magnusson A. Assessment of tibial torsion employing fluoroscopy, computed tomography and the cryosectioning technique. Acta Radiol 1989; 30: 75-80

5. Jakob RP, Haertel M, Stussi E. Tibial torsion calculated by computerised tomography and compared to other methods of measurement. J Bone Joint Surg Br 1980; 62-B: 238-242

6. Jend HH, Heller M, Dallek M, Schoettle H. Measurement of tibial torsion by computer tomography. Acta Radiol Diagn (Stockh) 1981; 22: 271-276

7. Lee SH, Chung CY, Park MS, Choi IH, Cho TJ. Tibial torsion in cerebral palsy: validity and reliability of measurement. Clin Orthop Relat Res 2009; 467: 20982104

8. Weiner DS, Cook AJ, Hoyt WA Jr, Oravec CE. Computed tomography in the measurement of femoral anteversion. Orthopedics 1978; 1: 299-306

9. King HA, Staheli LT. Torsional problems in cerebral palsy. Foot Ankle 1984; 4: 180-184

10. Song DH, Eun BL, Park SH, Lee JY, Tockgo YC. Tibial torsion in children of the Jeju area. Korean J Pediatr 2005; 48: 75-80
11. Jang SH, Woo BS, Park SB, Lee SG. Relationship between femoral anteversion and tibial torsion in intoeing gait. J Korean Acad of Rehab Med 1999; 23: 390396

12. LaGasse DJ, Staheli LT. The measurement of femoral anteversion. A comparison of the fluoroscopic and biplane roentgenographic methods of measurement. Clin Orthop Relat Res 1972; 86: 13-15

13. Mahboubi S, Horstmann H. Femoral torsion: CT measurement. Radiology 1986; 160: 843-844

14. Billing L. Roentgen examination of the proximal femur end in children and adolescents; a standardized technique also suitable for determination of the collum-, anteversion-, and epiphyseal angles; a study of slipped epiphysis and coxa plana. Acta Radiol Suppl 1954; 110: 1-80

15. Rethlefsen SA, Healy BS, Wren TA, Skaggs DL, Kay RM. Causes of intoeing gait in children with cerebral palsy. J Bone Joint Surg Am 2006; 88: 2175-2180

16. Sass P, Hassan G. Lower extremity abnormalities in children. Am Fam Physician 2003; 68: 461-468

17. Staheli LT. Rotational problems in children. Instr Course Lect 1994; 43: 199-209 